\title{
Molecular regulation of S100P in human lung adenocarcinomas
}

\author{
GRIT REHBEIN ${ }^{1}$, ANDREAS SIMM ${ }^{1}$, HANS-STEFAN HOFMANN ${ }^{2}$, \\ ROLF-EDGAR SILBER $^{1}$ and BABETT BARTLING ${ }^{1}$
}

\begin{abstract}
${ }^{1}$ Department of Cardio-thoracic Surgery, University Hospital Halle (Saale), Martin Luther University Halle-Wittenberg, Halle (Saale); ${ }^{2}$ Department of Thoracic Surgery, Hospital Barmherzige Brüder Regensburg, Regensburg, Germany
\end{abstract}

Received February 19, 2008; Accepted April 10, 2008

\begin{abstract}
Lung adenocarcinoma is characterised by an upregulation of S100P, which mediates its function intracellularly but also extracellularly. Recent studies suggest that extracellular S100P contributes to tumour development following interaction with the receptor for advanced glycation end-products (RAGE). As RAGE is highly downregulated in lung cancer, one might speculate that S100P supports tumorigenesis via other pathways. Here, we showed that S100P primarily localises in the cytoplasmic and nuclear region as determined for lung adenocarcinoma specimens (immunohistochemistry) and H358 lung adenocarcinoma cells stably overexpressing S100P (GFP-S100P; fluorescence microscopy). $\mathrm{S} 100 \mathrm{P}$ overexpression in $\mathrm{H} 358$ cells induced a more frequent formation of tumour colonies in vitro (soft agar assay). However, the S100P-overexpressing cell colonies showed a smaller colony size. This observation was supported by proliferation assays demonstrating a reduced proliferation per increasing cell density of the H358 cells overexpressing S100P. Migration of S100P-overexpressing H358 cells was diminished (transwell migration and in vitro wound scratch assays). Inhibiting DNA methylation with 5-aza-2'deoxycytidine enhanced the mRNA expression of S100P, whereas high S100P levels following overexpression suppressed the mRNA expression of endogenous S100P. In this regard, an upregulation of S100P in lung adenocarcinomas was only determined for early/T1 stage but not more advanced/T2 stage tumours compared with normal lung tissues. Thus, S100P induction may be considered an important step in the initial stage of lung adenocarcinomas, whereas its downregulation in advanced stages seems to be important for tumour progression in which DNA methylation and/or feedback transcription processes play a critical role.
\end{abstract}

Correspondence to: Dr Babett Bartling, Klinik für Herz- und Thoraxchirurgie, Universitätsklinikum Halle (Saale), Ernst-GrubeStr. 40, D-06120 Halle (Saale), Germany

E-mail: babett.bartling@medizin.uni-halle.de

Key words: lung cancer, S100P, staging, RAGE

\section{Introduction}

Lung cancer accounts for the most common type of cancerinduced mortality worldwide (1), and patient survival highly depends on early recognition of the disease. Approximately $75 \%$ of all lung malignancies belong to the heterogeneous group of non-small cell lung carcinomas (NSCLCs), which is broadly classified into squamous cell lung carcinomas and lung adenocarcinomas (2). Although diagnosis and histological classification are still based on clinicopathological features, advances in molecular-genetic classification will likely lead to improvements in the identification and therapy of patients with lung cancer. In this context, it has been shown that the expression of defined genes reliably distinguishes tumour from normal lung tissues and squamous cell carcinomas from adenocarcinomas $(3,4)$.

$\mathrm{S} 100 \mathrm{P}$ is one of the genes upregulated in the early tumour stage of lung cancer patients $(5,6)$. Our recent studies additionally demonstrated that the elevated expression of $\mathrm{S} 100 \mathrm{P}$ is associated with lung adenocarcinomas, whereas squamous cell lung carcinomas did not show highly altered S100P levels compared with normal lung (6). However, an upregulation of S100P has not only been reported for tumour tissues of the lung but also for breast (7-9), pancreas (10-13), prostate $(14,15)$ and colon $(16,17)$. Moreover, doxorubicinresistant cancer cell lines are characterised by an increased expression of S100P indicating the involvement of S100P in drug-resistance processes (18).

S100P belongs to the S100 family of calcium-binding proteins. It was first purified from human placenta S100P (19), but it is also expressed in other organs (20). S100 proteins are low-molecular-weight proteins that form homodimers but also heterodimers via two distinct calciumbinding EF-hand motifs (21-23). In the case of S100P, heterodimers are described with S100A1 (24), S100A6 (25) and S100Z (26). Although the precise function of S100P and other S100s is not well understood, it is assumed that these proteins mediate their action after dimerisation via binding to and regulating the activity of distinct intracellular effector molecules. In this context, is has been shown that S100P can interact with and partially activate ezrin (27), which modulates the state of actin filaments via direct linkage between actin filaments and the plasma membrane (28). Therefore, S100Pezrin interactions might contribute to processes at the membrane-cytoskeleton interface such as the regulation of 
cell migration. The potential role of S100P in cell migration is supported by the observation that $\mathrm{S} 100 \mathrm{P}$ expression is associated with the metastasis of cancer cells in vitro $(5,29,30)$ and in vivo $(5,8)$. Although the cellular function is unclear, S100P binding has been reported for other proteins including Cacy/SIP, a component of a novel pathway of ß-catenin ubiquitination (31) and its homologue Sgt1 (32). In addition to the intracellular function, S100P exerts its biological effect at extracellular sites after active release from the cell (33). S100P binding to the multi-ligand receptor for advanced glycation end-products (RAGE) and stimulation of the extracellular signal-regulated kinases (ERK)-1/-2 and nuclear factor (NF)- $\mathrm{\kappa B}$ are described as potential downstream mechanisms $(30,33)$.

The dual, intracellular and extracellular, protein function is characteristic for other proteins of the S100 family (34). Despite the potential importance of RAGE in mediating the biological function of S100P, it must be considered that interactions of S100P with other molecules or not yet identified molecules could play a more pivotal role in the S100P-dependent action of tumour cells. Moreover, we demonstrated a strong downregulation of RAGE in early and advanced NSCLCs independent of the histopathological subtype $(35,36)$ which challenges the primary role of RAGE in lung carcinogenesis. As S100P expression was mainly associated with lung adenocarcinoma tissues (6), our experimental study focused on the importance of S100P in the development of lung adenocarcinomas and its relation to the concurrent downregulation of RAGE.

\section{Materials and methods}

Patients. We examined lung specimens from tumour and paired non-tumour tissue of 16 patients with lung adenocarcinoma, who underwent pulmonary resection surgery. Patients were grouped according to the primary tumour size into $\mathrm{T}$ stage 1 to 4 (37). The use of human tissue was approved by the local ethics committee (Medical Faculty, Halle/Saale, Germany).

RNA expression analysis. Total RNA of lung and paired tumour tissue specimens was extracted by standard guanidinium isothiocyanate/caesium chloride centrifugation. The mRNA expression of $\mathrm{S} 100 \mathrm{P}$ was analysed by real-time polymerase chain reaction (PCR) per external cDNA standards as described recently (6). The total RNA of cultured cells was isolated in accordance to the protocol of the RNeasy Mini Kit (Qiagen, Hilden, Germany) and reverse transcribed into cDNA by use of the Superscript II $^{\mathrm{TM}}$ reverse transcriptase (Invitrogen, Carlsbad, CA, USA). Thereafter, cDNA was amplified by PCR with 5 pmol of each primer and $1 \mathrm{X}$ complete PCR master mix (Promega, Mannheim, Germany). For detection of total (endogenous plus overexpressed) S100P mRNA the following primer pair was used: 5'CAT GGT CCT GCT GGA GTT CGT G 3' (sense) and 5'AAA GGA TCC TCA TTT GAG TCC TGC 3' (antisense). The endogenous (non-overexpressed) level of S100P mRNA was analysed by another pair of primers: 5'ATC TAG CAC CAT GAC GGA AC 3' (sense) and 5'AGC CTA GGG GAA TAA TTG CC 3' (antisense), which can hybridise to a cDNA sequence outside the coding region of S100P (NM_005980). Exact RNA loading per cDNA synthesis was determined by PCR amplification of $18 \mathrm{~S}$ rRNA with specific primers: 5'GTT GGT GGA GCG ATT TGT CTG G 3' (sense) and 5'AGG GCA GGG ACT TAA TCA ACG C 3' (antisense). The optical intensity of all amplified PCR fragments was evaluated after agarose gel-electrophoresis by use of the LAS3000 computer-based imaging system (FujiFilm, Tokyo, Japan) equipped with AIDA3.5 software (Raytest, Straubenhardt, Germany). Expression data were normalised per amplification of $18 \mathrm{~S}$ rRNA.

Construction of expression vectors and transfection. The coding sequence of S100P (NM_005980) was amplified from human cDNA by PCR and cloned into the mammalian expression vector pEGFP-C1 (BD Biosciences Clontech, Heidelberg, Germany) which generates GFP-S100P. Moreover, the cDNAs of full-length human RAGE (XM_004205) or $\Delta$ cytoRAGE (without cytoplasmic domain) were generated by PCR and cloned into the pcDNA3.1. vector system (BD Bioscience Clontech). Each plasmid vector was controlled by DNA sequencing using the BigDye ${ }^{\circledR}$ Terminator v.1.1 Cycle sequencing kit (AB Applied Biosystems, Warrington, UK). Recombinant plasmids or the vector alone were transfected into the respective cell line using Effecten transfection reagent (Qiagen). For stable expression, cells were selected for Geneticin resistance $(\mathrm{G} 418,1 \mathrm{mg} / \mathrm{ml}$; BD Biosciences Clontech) and/or for green fluorescent protein (GFP) fluorescence using the FACSVantage cell sorter (BD Biosciences, San Diego, CA, USA). Altogether, cells with or without overexpression of GFP-S100P and cells with or without co-overexpression of either RAGE or $\Delta$ cytoRAGE were generated.

Cell culture conditions. We studied the lung adenocarcinoma cell line H358 and the pancreas carcinoma cell line Panc-1 (ATCC, Manassas, VA, USA). All cells were cultured in Dulbecco's modified Eagle's medium (DMEM) supplemented with $10 \%$ fetal calf serum (FCS) (Invitrogen, Karlsruhe, Germany) at $37^{\circ} \mathrm{C}$ in a humidified $10 \% \mathrm{CO}_{2}$ atmosphere. In certain experiments conditioned medium was used, which was taken after $24 \mathrm{~h}$ from confluent $\mathrm{H} 358$ cells and internally normalised per number of cells. Cell death was induced following treatment of the cells with chemotherapeutics: $30 \mu \mathrm{M}$ cisplatin (Medac, Hamburg, Germany), $10 \mu \mathrm{M}$ etoposide (Vepesid $^{\circledR}$, Bristol-Meyers Squibb, Munich, Germany) and $5 \mathrm{nM}$ paclitaxel (Merck, Bad Soden, Germany). Moreover, 5aza-2'deoxycytidine (Sigma, Deisenhofen, Germany), an inhibitor of DNA methylation, was applied at $10 \mu \mathrm{M}$ for one to three days and replenished on a daily basis.

Cell proliferation and growth studies. For cell proliferation assay, cells were seeded at a density of $1.3 \times 10^{4}$ cells per $\mathrm{cm}^{2}$ for seven days in DMEM containing 10\% FCS, which was regularly changed. The cell proliferation was determined daily by counting the total number of living cells using the Casy ${ }^{\circledR}$ Cell Counter system (Schärfe System GmbH, Reutlingen, Germany), and the population doubling (PD) of cells was calculated from the equation: (ln cell number day $x_{x}-1 n$ cell number $\left._{\text {day } x-1}\right) / \ln 2$ (38). Tumour colony-forming assay was 
performed with $2 \times 10^{4}$ cells $/ \mathrm{ml}$ agar $(0.6 \%$ in DMEM supplemented with $10 \%$ FCS) according to a standard protocol (39). The number and diameter of the cell colonies were evaluated after 14 days by microscopy using the Zeiss Axiovert microscope equipped with a Spot RT camera (Zeiss, Jena, Germany) and Metamorph4.6.5. software (Visitron Systems, Puchheim, Germany). The diameter of 100 randomly selected colonies was estimated and averaged.

In vitro migration. Cell migration was analysed by use of the in vitro wound scratch model. Briefly, scratches were placed into a monolayer of confluent cells by a pipette tip to generate defined cell-free areas. To prevent the proliferation of the cells into the cell-free area of the scratch, the cells were then cultured in FCS-reduced conditions (DMEM supplemented with $1 \%$ FCS). The distances between the two edges of the migrating cells were regularly estimated using a Zeiss Axiovert microscope equipped with a Spot RT camera and Metamorph4.6.5. software. Three distances per scratch were always measured and finally averaged. Additionally, we studied transwell cell migration by use of cell culture inserts of $8-\mu \mathrm{m}$ pore size (ThinCerts, Frickenhausen, Germany). Cells $\left(5 \times 10^{4} / \mathrm{cm}^{2}\right)$ were seeded into the insert containing FCS-free DMEM. The insert was then transferred into a larger cell culture chamber which contained DMEM with $10 \%$ FCS to stimulate cell migration. After six days of culturing in this serum gradient, non-migrated cells were removed from the inner site of the insert by a pad of cotton wool. The number of migrated cells was estimated by Alamar Blue $^{\mathrm{TM}}$ staining according to the manufacturer's instructions (BioSource, Solingen, Germany).

Cell death analysis. Apoptotic cell death was analysed after $48 \mathrm{~h}$ of treatment with defined chemotherapeutics by investigating the nuclear alterations. Treated cells were resuspended in $70 \%$ ethanol, spread on slides and allowed to air-dry. After staining with Hoechst $33342(1 \mu \mathrm{g} / \mathrm{ml}$ in PBS; Molecular Probes Europe, Leiden, The Netherlands), 300 cells of each sample were assessed by fluorescence microscopy (Axiovert microscope, Zeiss) and scored as the percentage of cells with fragmented or condensed nuclear morphology. Cell death indicated by necrotic or late-apoptotic features was measured by the uptake of propidium iodide $(5 \mu \mathrm{g} / \mathrm{ml}$ in PBS; Sigma) and subsequent cytometric analysis using the FACSCalibur flow cytometer equipped with CellQuest Pro software (BD Biosciences).

Protein expression analysis. Proteins were extracted in SDS lysis buffer [10 mM Tris-(hydroxymethyl)-aminomethane, $2 \%$ sodium dodecyl sulfate, protease inhibitor mix (Sigma, Deisenhofen, Germany); $\mathrm{pH}$ 7.4)], and the protein concentration was measured by the bicinchoninic acid assay (Pierce, Rockford, IL, USA). Nuclear and cytoplasmic protein from H358 cells was prepared as described recently (6). Protein samples were subjected to standard immunoblot procedure and incubated with the respective primary antibody: mouse monoclonal anti-GFP (Roche Diagnostics, Mannheim, Germany) or anti-S100P (BD Biosciences Transduction Laboratory, San Diego, CA, USA) or rabbit polyclonal antiGAPDH (Abcam, Cambridge, UK). Bound antibodies were detected by horseradish peroxidase-conjugated secondary antibodies and subsequent ECLPlus reagent (Amersham, Buckinghamshire, UK). The intensities of all visualised signals were densitometrically quantified by use of the LAS3000 computer-based imaging system (FujiFilm) equipped with AIDA3.5 software (Raytest). Moreover, we determined the secretion of S100P from H538 cells overexpressing GFPS100P compared with H358 control cells overexpressing GFP alone. Confluent cells were cultured in DMEM without phenol-red dye containing 5\% FCS. The conditioned medium was removed after one day of culture, normalised by the number of cells and the GFP-specific fluorescence was monitored in duplicate for each medium sample by use of the Fluostar Optima reader (BMG Labtechnologies, Offenburg, Germany) at $485 \mathrm{~nm}$ excitation and $520 \mathrm{~nm}$ emission.

Fluorescence microscopy and immunohistochemistry. S100P detection was performed according to the GFP fluorescence of cells overexpressing GFP-S100P. Cells were fixed in $4 \%$ PBS-buffered formaldehyde. In addition, immunostaining of cells was performed by use of the mouse monoclonal RAGE antibody according to the recently described protocol (36). After co-staining of the nuclei with 4',6-diamidino-2phenylindole dihydrochloride (DAPI) solution $(1 \mu \mathrm{g} / \mathrm{ml}$ in PBS; Molecular Probes), slides were embedded in glycergel mounting medium (DakoCytomation, Hamburg, Germany) and analysed by fluorescence microscopy (Axiovert microscope equipped with RT Spot camera, Zeiss). Immunohistochemistry of S100P was performed by use of the mouse antiS100P antibody (BD Biosciences Transduction Laboratory; 1:400 in PBS) as described recently (6).

Data analysis. Patient data were presented as the median. Box and Whisker plots were constructed for illustration: the line within the box marks the 50th percentile (median), boundaries of the box indicate the 25th and 75th percentiles, and Whiskers above and below the box represent the 5th and 95th percentiles. Results of the experimental analyses were either presented as absolute data or were calculated as relative changes per internal control. Statistical analyses were performed with the standard ANOVA procedure or ANOVA on Ranks procedure in the case of non-parametric data (SigmaStat software; Jandel Corp., San Rafael, CA, USA). All data of the experimental assays were reported as the mean \pm $\mathrm{SEM}$ or $\mathrm{SD}$, and $\mathrm{p} \leq 0.05$ was accepted as indicating a significant difference in mean values.

\section{Results}

S100P expression in lung adenocarcinoma cells. Lung adenocarcinoma specimens were characterised by an increased mRNA level of S100P compared with normal lung tissues (Fig. 1). Detailed analyses revealed that this S100P upregulation was related to lung adenocarcinomas of the tumour stage T1 but not to T2 or T3-4 stage adenocarcinomas (Fig. 1). T stages 1 and 2 were dependent on the size and not the pulmonary location of the tumour. The lung adenocarcinoma cell line $\mathrm{H} 358$ also showed a detectable mRNA amount of S100P (Fig. 2A). Inhibition of DNA methylation by treating cells with 5-aza-2'deoxycytidine (5- 


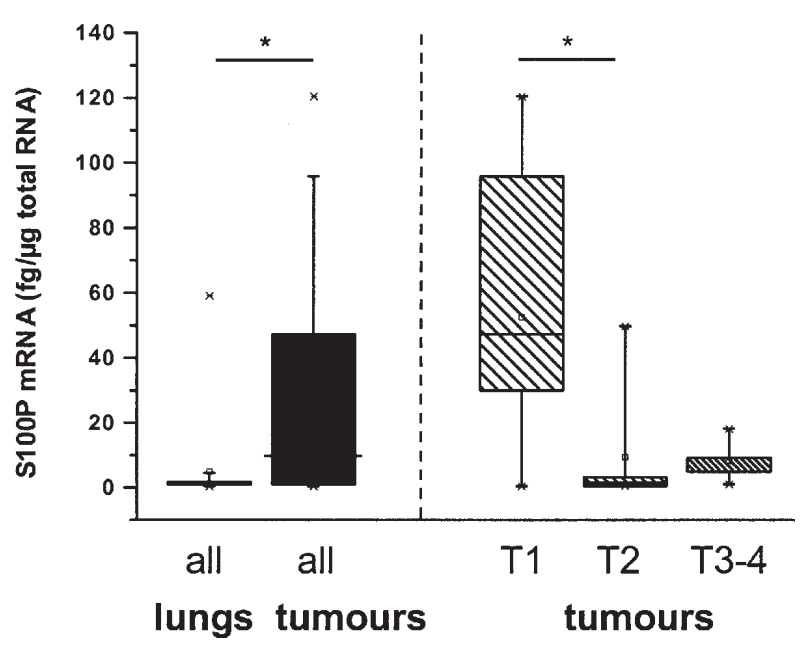

Figure 1. The mRNA level of S100P in normal lung tissue and lung adenocarcinoma specimens dependent on tumour stages: T1 (tumour $\leq 3 \mathrm{~cm}$ in maximal diameter; $\mathrm{n}=7$ ), $\mathrm{T} 2$ (tumour $>3 \mathrm{~cm}$ in maximal diameter; $\mathrm{n}=6$ ) and T3-4 (tumour infiltrating local and distant tissues, respectively; $n=4$ ). Expression analyses were performed by real-time PCR per standard S100P cDNA molecules. Data are represented as box and Whisker plots with ${ }^{*} \mathrm{p}<0.05$.

aza-2'DC) enhanced the mRNA expression of S100P in H358 cells after two days of treatment, which was normalised after three days (Fig. 2A). Therefore, H358 cells were stably transfected with S100P that had been N-terminally tagged with GFP (GFP-S100P; Fig. 2B). As we studied the GFPtagged S100P in all experiments, this fusion protein was commonly named S100P. Stable overexpression of S100P caused an accordingly high level of total S100P mRNA in H358 cells but suppressed the level of endogenously expressed S100P mRNA (Fig. 2C).

Microscopic analyses showed the localisation of S100P in the cytoplasmic and nuclear region of H358 cells (Fig. 3A). Although this observation was confirmed by immunoblot studies of the respective cytoplasmic and nuclear protein fractions, the relative amount of nuclear S100P in H358 cells was not that obvious as determined by microscopy (Fig. 3B). This might be based on a weak interaction of S100P with the outer nuclear membrane or on the release of nuclear S100P during the preparation of purified nuclei. However, immunohistochemistry of a lung adenocarcinoma specimen, which was selected because of its high S100P mRNA level, confirmed the nucleo-cytoplasmic distribution of S100P in situ (Fig. 3C). Analysis of the GFP fluorescence in conditioned cell culture media did not find a detectable amount of extracellular S100P for H358 cells overexpressing the GFP-tagged S100P compared with control cells overexpressing GFP alone (data not shown). Immunocytochemistry with subsequent fluorescence microscopy of H358 cells co-overexpressing RAGE also did not indicate the primary interaction of S100P with RAGE at the cell surface (Fig. 3A).

Influence of S100P on tumour cell proliferation and growth in vitro. $\mathrm{H} 358$ cells overexpressing $\mathrm{S} 100 \mathrm{P}$ were characterised by an increased capacity to form cell colonies from a single H358 cell in a soft agar colony-forming assay (Fig. 4A).
A

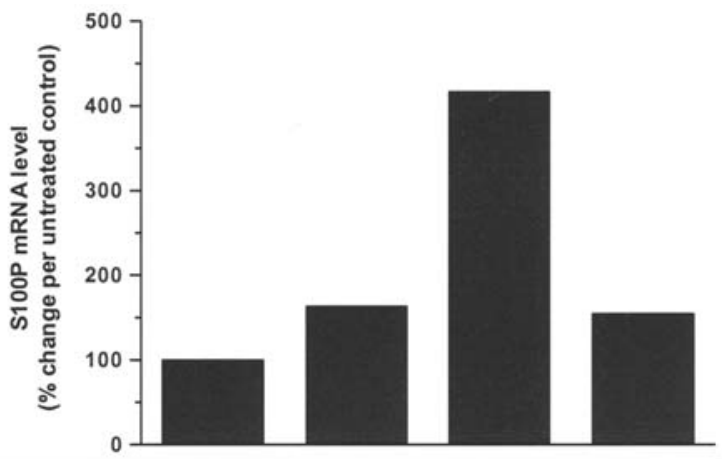

\begin{tabular}{|c|c|c|c|}
\hline 5-aza-2 DC & untreated 1d & $2 d$ & $3 d$ \\
\hline S100P mRNA & 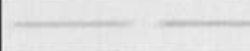 & & \\
\hline 18S rRNA & 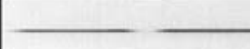 & & \\
\hline
\end{tabular}

B

\begin{tabular}{|c|c|c|}
\hline H358 & control & S100P \\
\hline S100P & - & $=$ \\
\hline GAPDH & thesters & $=$ \\
\hline
\end{tabular}

C

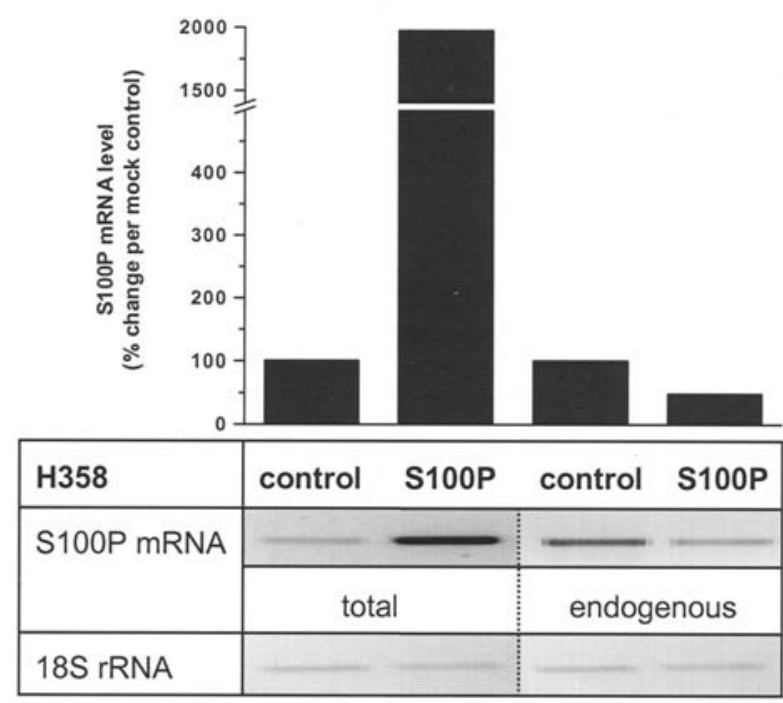

Figure 2. (A) mRNA level of S100P in H358 lung adenocarcinoma cells treated with 5-aza-2'deoxycytidine (5-aza-2'DC; $10 \mu \mathrm{M}$ ) for one to three days. Two time-independent experiments showed the 5-aza-2'DC-induced expression of S100P mRNA, which was calculated as percent changes per untreated control and averaged. (B) Immunoblot analyses indicate the protein expression level of S100P in $\mathrm{H} 358$ cells stably transfected with S100P (N-terminally tagged with GFP; GFP-S100P) compared with vectortransfected cells (control). (C) PCR analyses with divergent primers determined the mRNA level of either total (endogenous plus overexpressed) or only endogenous (non-overexpressed) S100P.

However, the final size of the cell colonies was significantly reduced in this three-dimensional assay for the S100P-overexpressing H358 cells (Fig. 4B). Co-overexpressing RAGE did not affect the S100P-related impact on the colony formation and size (Fig. 4A and B). In accordance with the 
A

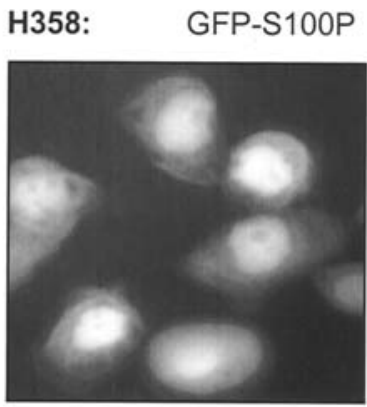

H358-RAGE: GFP-S100P
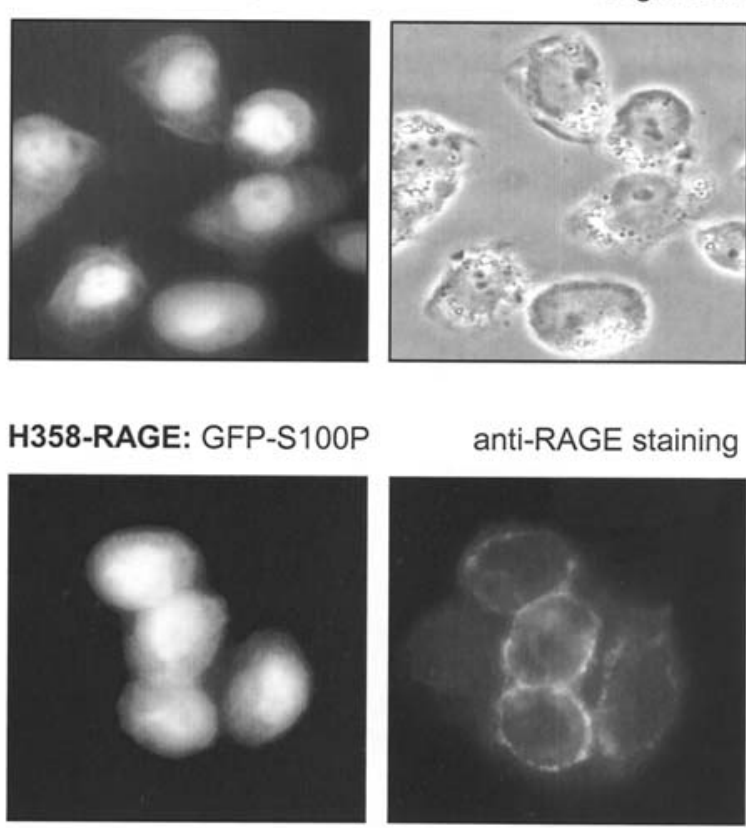

anti-RAGE staining

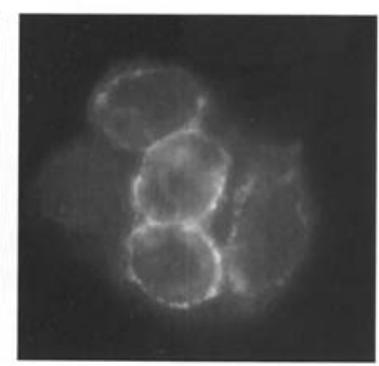

B

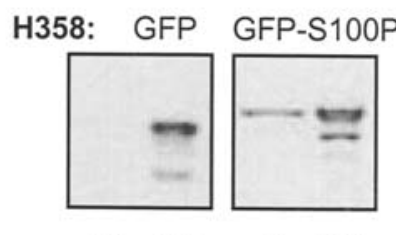

N CP

N CP

C

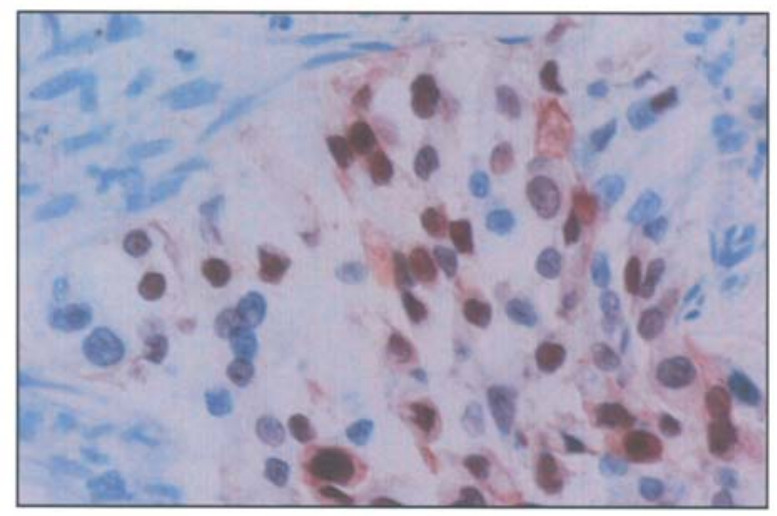

lung adenocarcinoma:

anti-S100P staining

nuclei (haematoxylin) staining

Figure 3. (A) Fluorescence microscopy of H358 lung cancer cells overexpressing GFP-tagged S100P (GFP-S100P) or co-overexpressing GFP-S100P and RAGE indicates the nucleo-cytoplasmic distribution of S100P. (B) Anti-GFP immunoblot analyses of the nuclear (N) and cytoplasmic (CP) protein fraction from H358 cells overexpressing GFPtagged S100P. (C) In situ cellular localisation of S100P is shown by immunohistochemistry of a selected lung adenocarcinoma section.

final size of the formed cell colonies in vitro, H358 cells overexpressing S100P were characterised by a lower cell population doubling (PD) per increasing cell density in standard cell culture dishes in comparison to vector- transfected (control) H358 cells (Fig. 4C). Plotting the number of $\mathrm{H} 358$ cells per time of cells in culture emphasized reduced cell proliferation following S100P overexpression (Fig. 4D). In contrast to the three-dimensional soft-agar model, stably co-overexpressing RAGE abolished the S100Prelated impact on cell proliferation in the two-dimensional culture model (Fig. 4D). The RAGE-dependent effect was confirmed by $\mathrm{H} 358$ cells stably transfected with a mutant of RAGE ( $\Delta$ cytoRAGE; RAGE without intracellular domain), which were also characterised by a reduced cell proliferation in response to $\mathrm{S} 100 \mathrm{P}$ overexpression (data not shown). The susceptibility of H358 cells to selected chemotherapeutics (cisplatin, etoposide, paclitaxel) was not impaired but slightly enhanced by overexpression of S100P (Fig. 5A and B).

Influence of S100P on cell migration. In vitro wound scratch assays showed a reduced migration of $\mathrm{H} 358$ cells overexpressing S100P (Fig. 6A). The relative reduction in migration was also obvious for $\mathrm{H} 358$ cells co-overexpressing RAGE (Fig. 6B). The S100P-related impact on H358 cell migration was confirmed by studying these cells in a transwell migration assay (Fig. 6C). As it was recently demonstrated that the migration of the pancreas carcinoma cell line Panc-1 was enhanced following S100P overexpression (29), we generated Panc-1 cells stably transfected with S100P. Indeed, these cells showed an increased cell migration in the transwell migration assay, but the intracellular localisation of S100P (Fig. 6C) and the non-release of S100P (data not shown) from Panc-1 cells were comparable with $\mathrm{H} 358$ cells.

\section{Discussion}

Expression analyses of tumour specimens from patients with non-small cell lung carcinoma (NSCLC) clearly showed that the cancer-related upregulation of S100P is mainly associated with lung adenocarcinoma (6). In correspondence with our findings in the human lung, the aberrant expression of S100P has also been described for adenocarcinoma tissues of the pancreas $(11,13)$ and colon $(16)$. One of the molecular alterations responsible for the induced expression of S100P in cancer cells might be the hypomethylation in $5^{\prime} \mathrm{CpG}$ islands of the S100P gene region as recently identified for pancreatic and prostate cancer $(12,40)$. Inhibiting DNA methylation with 5-aza-2'deoxycytidine (5-aza-2'DC) induced the mRNA expression of S100P in lung cancer cells (H358) indicating the importance of DNA methylation processes in the transcriptional regulation of S100P in lung tumours as well. However, the continuously high level of S100P seems to cause a negative feedback mechanism since the mRNA level of endogenous S100P was diminished in H358 cells stably overexpressing S100P. This assumption can be supported by our observation that the 5-aza-2'DC-induced mRNA expression of S100P after two days was normalised one day later. As S100P is only upregulated in stage T1 tumours but unchanged in T2 and T3-4 tumours, the transcriptional control by negative feedback loop processes could be a potential mechanism responsible for the tumour stagedependent S100P expression.

To investigate the importance of S100P in the pathogenesis of lung adenocarcinomas, we overexpressed S100P in the 
A

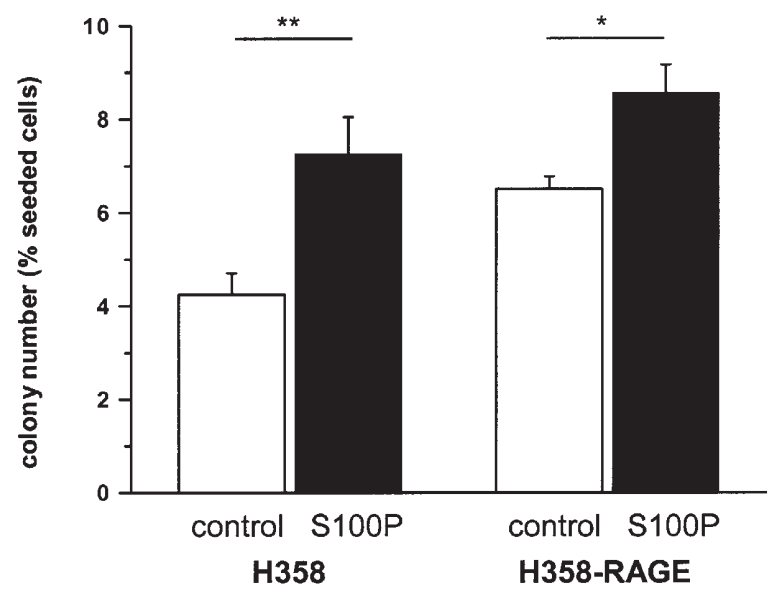

B

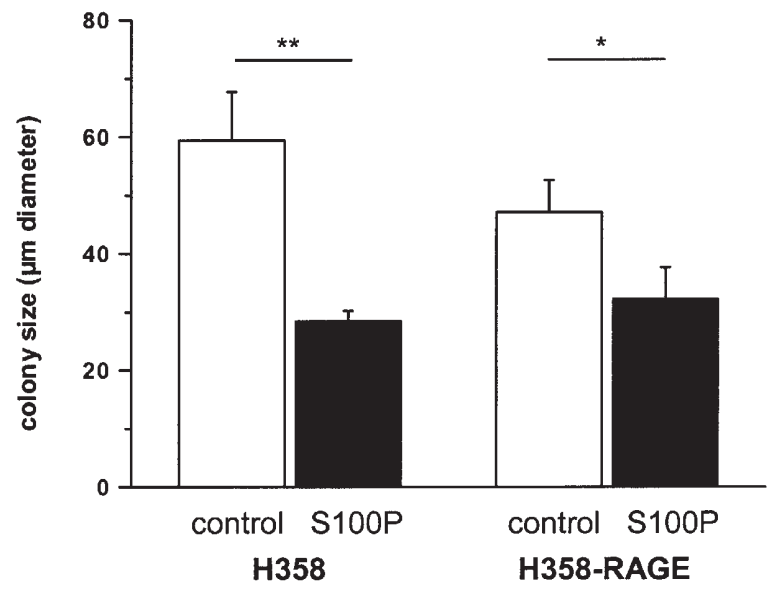

C

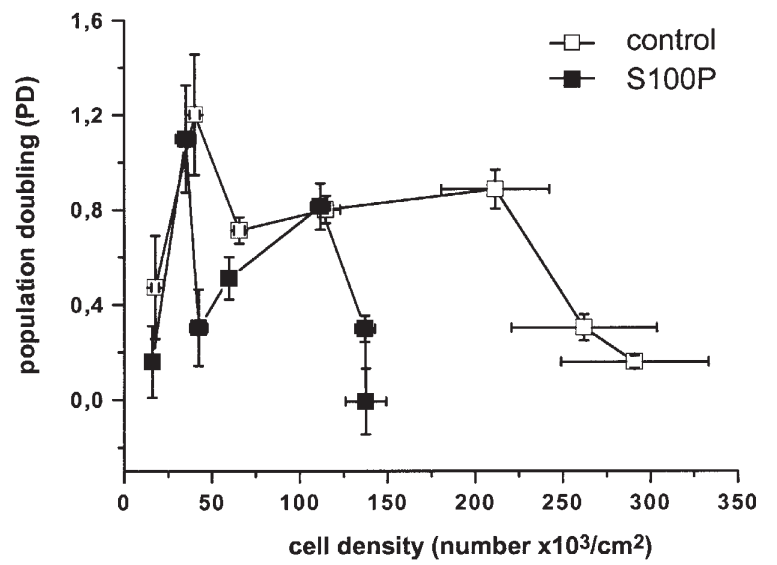

D

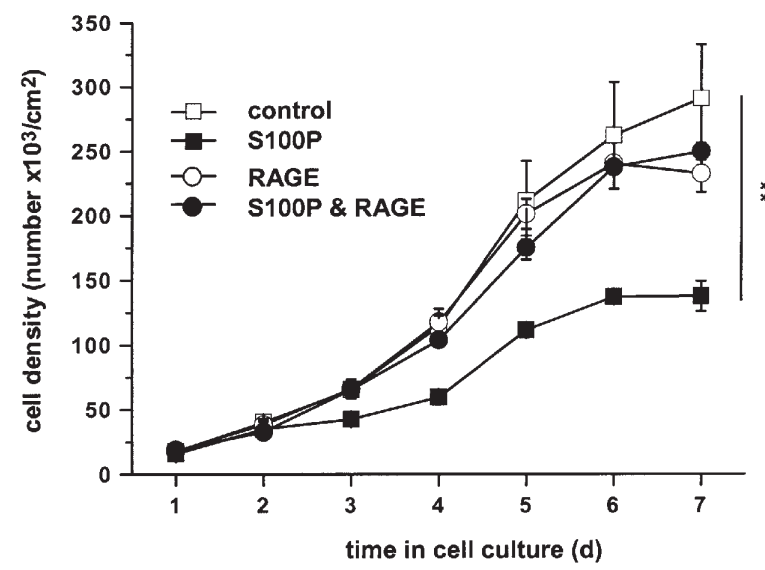

Figure 4. (A) Tumour colony formation of H358 cells overexpressing S100P compared with vector-transfected cells (control) in a soft agar assay. In addition, H358 cells with and without co-overexpression of RAGE were analysed. The number of formed cell colonies ( $>20 \mu \mathrm{m}$ diameter) and (B) size of these colonies were estimated by microscopy. (C) Proliferation of H358 cells overexpressing S100P was measured per vector-transfected cells (control) for a time period of seven days. The population doubling (PD) was calculated and graphed per number of cells determined daily. Mean data \pm SEM are provided with ${ }^{*} \mathrm{p}<0.05$ and ${ }^{* *} \mathrm{p}<0.01(\mathrm{n}=6)$.

A

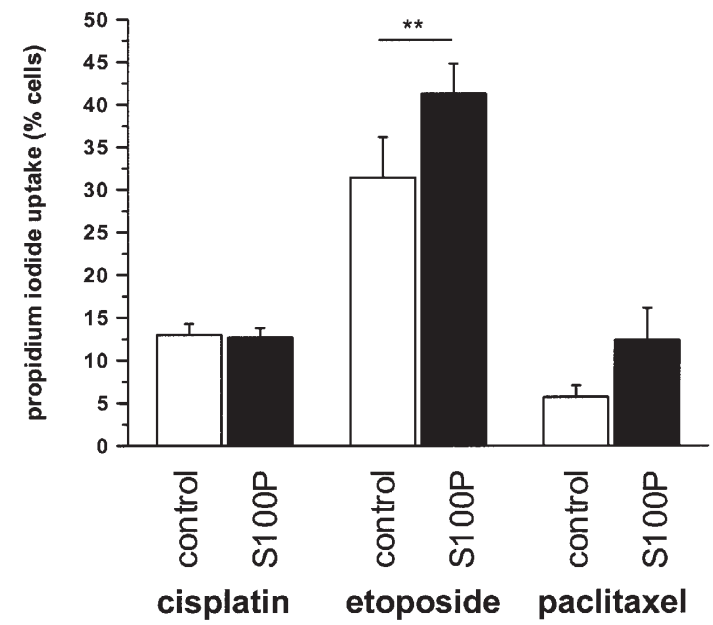

B

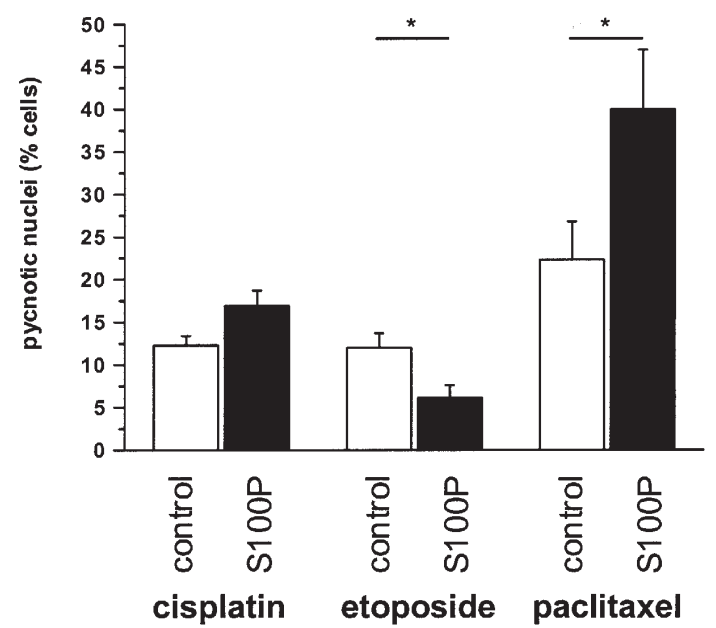

Figure 5. Chemotherapy-induced death of H358 cells overexpressing S100P compared with vector-transfected cells (control). Cell death was analysed for (A) necrotic (uptake of propidium iodide) and (B) apoptotic (pycnotic nuclei) features after $40 \mathrm{~h}$ of treatment with cisplatin $(30 \mu \mathrm{M})$, etoposide (10 $\mu \mathrm{M})$ or paclitaxel $(5 \mathrm{nM})$. Means \pm SEM are provided with ${ }^{*} \mathrm{p}<0.05$ and ${ }^{* *} \mathrm{p}<0.01(\mathrm{n}=6)$. 
A
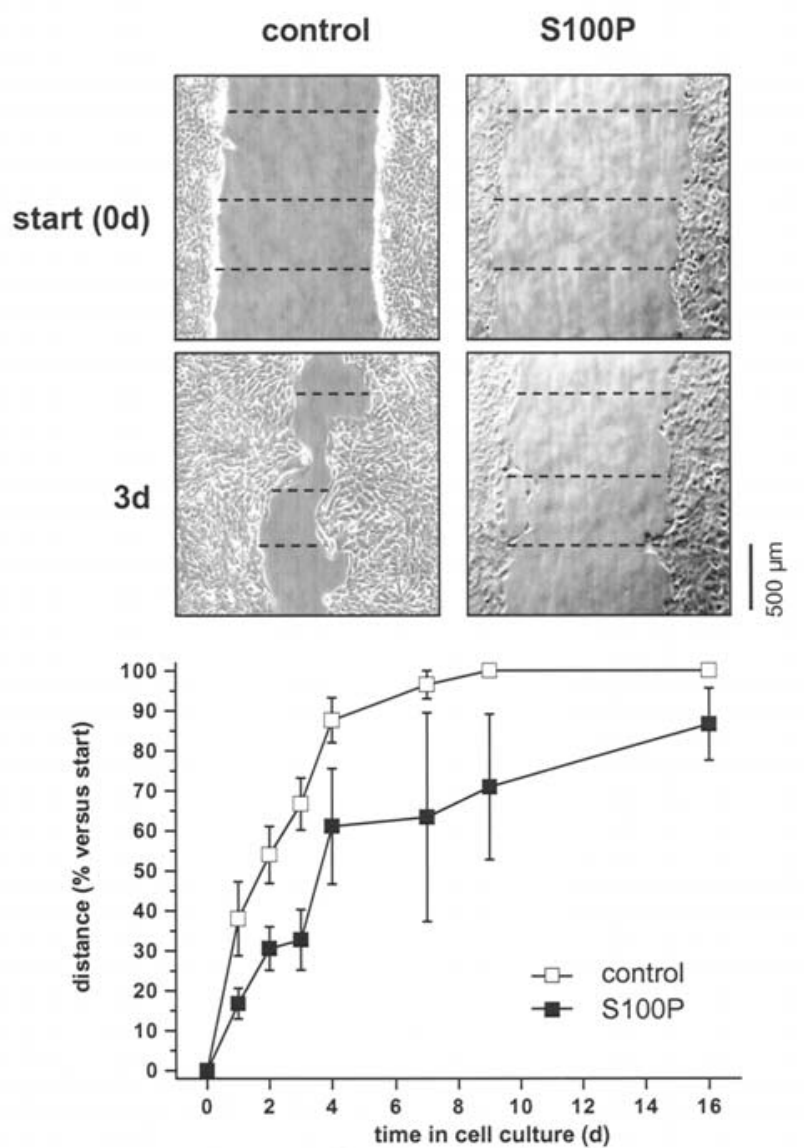

B

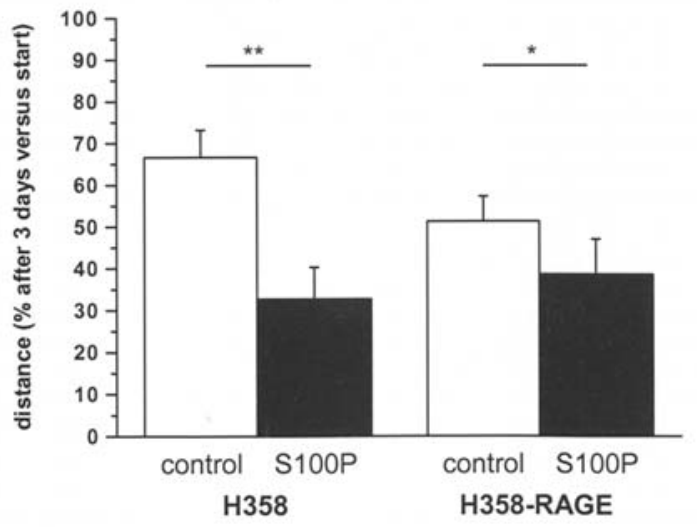

human lung cancer cell line $\mathrm{H} 358$, which is a frequently studied adenocarcinoma cell line with well-pronounced tumour growth in tumorigenicity assays in vitro and in vivo $(36,41)$. S100P was generated as a fusion protein N-terminally tagged with GFP because it allows an antibody-independent detection of intracellular and extracellular S100P. Moreover, GFP-tagged S100P protein was studied since Koltzscher et al $(29,30)$ observed the intact homodimer formation of this fusion protein with subsequent target protein interaction and intracellular relocation. These observations are supported by our analyses. Firstly, the reduced mRNA expression of endogenous S100P in H358 overexpressing the GFP-tagged $\mathrm{S} 100 \mathrm{P}$ indicates its recognition and specific response by the cell. Moreover, the intracellular localisation of GFP-tagged $\mathrm{S} 100 \mathrm{P}$ in H358 cells is comparable with the localisation of endogenous S100P in tumour cells of lung adenocarcinoma sections. Accordingly, S100P showed cytoplasmic and nuclear distribution in lung adenocarcinoma cells which was
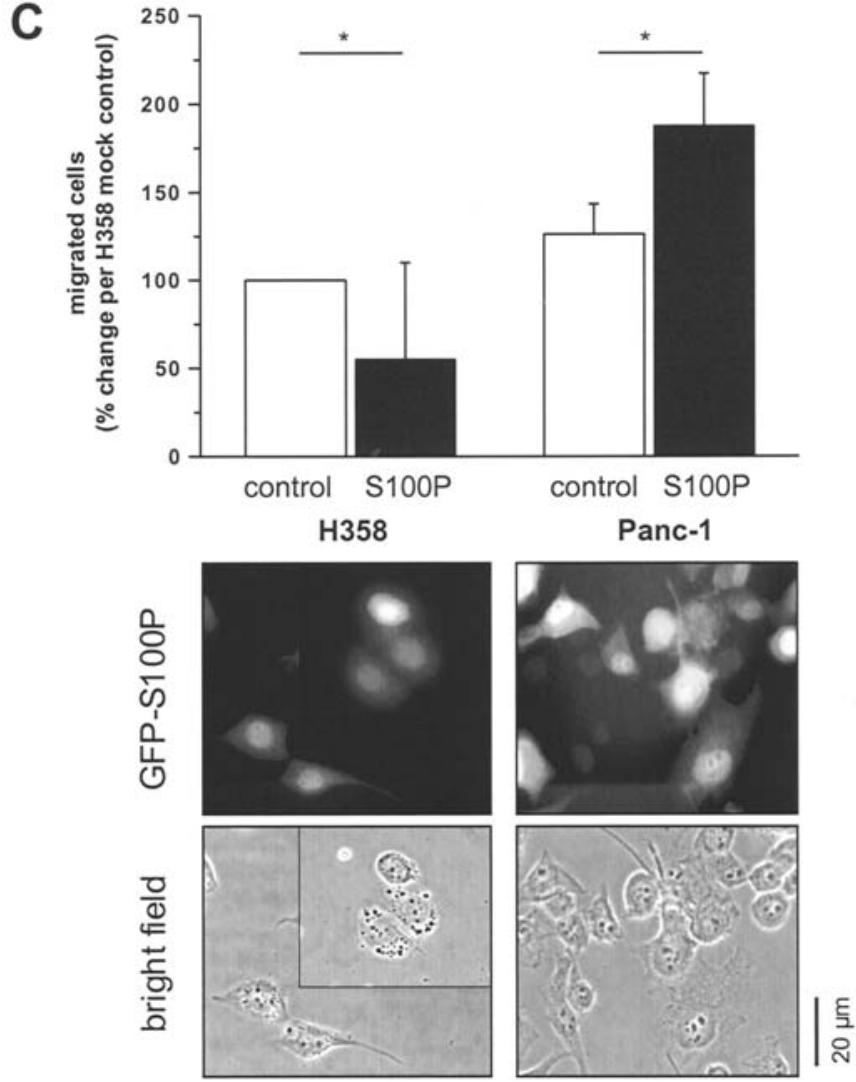

Figure 6. (A) Influence of S100P on the migration of H358 lung cancer cells in an in vitro wound scratch model. The distances between the two edges of the scratch were estimated and, finally, the migration of cells was calculated as a percentage of the adhered scratches for a time period of 16 days. (B) Additionally, cell migration data at day 3 are provided for $\mathrm{H} 358$ cells co-overexpressing S100P and RAGE. All data are means \pm SEM with * $\mathrm{p}<0.05(\mathrm{n}=4)$. (C) Transwell migration of carcinoma cell lines from lung (H358) and pancreas (Panc-1) overexpressing S100P compared with vectortransfected cells (control). Mean number of migrated cells \pm SEM was calculated as percent change per internal control cells. ${ }^{*} \mathrm{p}<0.05(\mathrm{n}=4)$. Fluorescence microscopy indicates the localisation of $\mathrm{S} 100 \mathrm{P}$ in both cell lines stably transfected with GFP-S100P.

previously described for prostate and pancreatic cancer cells $(13,14,42)$. The novel protein named S100P-binding protein S100PBPR was discovered as a nuclear interactive partner of $\mathrm{S} 100 \mathrm{P}$ (13), but the biological function of this interaction is still unknown.

The multi-ligand receptor RAGE was found to be a binding partner of extracellular S100P (33). Although S100P is detectable in normal lung tissue (20), the physiological level of S100P in the lung is rather low compared with the pathophysiological level in lung adenocarcinomas (6). On the other hand, RAGE is detectable at a very low level in lung adenocarcinomas but is highly expressed in normal lung $(35,36)$. For this reason, our study additionally focused on the combined alteration of both factors, S100P and RAGE, in lung adenocarcinomas. This study showed that the overexpression of S100P did not cause a detectable release of S100P into the extracellular space and co-localisation with RAGE in normal cell culture conditions. Nevertheless, intracellular S100P also 
indirectly influenced RAGE function by interfering with distinct RAGE-induced cell signalling pathways.

$\mathrm{S} 100 \mathrm{P}$ is believed to support the immortalisation of epithelial cells that finally leads to neoplastic transformation (7). As S100P is upregulated in pancreatic intraepithelial neoplasias $(13,43)$ as well as in early stage non-small cell lung carcinomas $(5,6)$, these in situ analyses additionally indicate the potential importance of S100P during tumour initiation. In this regard, our in vitro tumorigenicity assays demonstrated that the overexpression of S100P in H358 lung cancer cells results in a more frequent formation of tumour colonies from single cells cultured in soft agar. These data correspond to in vivo tumorigenicity assays showing a positive effect of S100P on the anchorage-independent growth of cells and tumour formation in immune-deficient mice $(29,44)$.

Although these findings indicate the tumorigenic potential of S100P, the three-dimensional growth/size of H358 tumour colonies in vitro was diminished in response to S100P overexpression. Additionally, two-dimensional cell proliferation assays showed a reduced proliferation of H358 cells overexpressing S100P per increasing cell density which suggests the activation of contact inhibition processes. These data are in contrast to findings of other studies, which demonstrated the overexpression of S100P as a promoter of cell proliferation and tumour growth in the case of pancreatic or cervical carcinoma cells $(29,44)$. One potential explanation might be molecular differences associated with the origin and subtype of the cells. Another explanation for our divergent findings could be differences in the basal expression of RAGE in cancer cells. In this context, we showed that the S100Pmediated impact on the cell proliferation was less pronounced in H358 cells co-overexpressing RAGE but not in cells cooverexpressing a deletion mutant of RAGE ( $\Delta$ cytoRAGE). This observation in turn supports experimental studies using pancreatic and colon cancer cells which indicated a positive effect of S100P on cancer cell proliferation via RAGE $(30,33)$. Although these data might suggest the contribution of the S100P-RAGE system in cell proliferation, it is speculative whether this system has significant importance in tumour growth because we did not observe RAGE-dependent effects for the three-dimensional growth/size of tumour cell colonies in vitro.

Accordingly, it must be noted that lung adenocarcinomas only showed an upregulation of S100P in early stage T1 tumours, whereas more advanced stage T2 tumours had no elevated levels of S100P. As S100P mediated a more frequent formation but less extensive growth of the H358 tumour cell colonies in vitro, one can conclude that $\mathrm{S} 100 \mathrm{P}$ plays an essential role in the initiation but a less essential or even an adverse role in the accelerated growth of lung adenocarcinomas. Therefore, the downregulation of S100P appears to be an important process allowing tumour extension. Negative feedback regulations and changes in the DNA methylation state are suggested as potential mechanisms that can impair the transcription level of S100P during tumour progression.

The cancer-induced mortality of NSCLC patients is predominantly caused by early metastasis. In this context, S100P has been shown to enhance the migration of lung cancer cells in vitro (5). Although this study used a lung cell line (HTB-58) derived from a squamous cell carcinoma, which is not characterised by a strong upregulation of S100P in situ (6), S100P-induced effects on cell migration have been described for other carcinoma cells $(29,30)$. Moreover, clinico-molecular analyses identified S100P as a predictor of metastasis and survival of cancer patients $(5,8,45)$. Similar to observations by Arumugam et al (29) and Fuentes et al (30), the overexpression of S100P promoted the migration of pancreatic carcinoma cells (Panc-1) in our experimental study. Although the interplay of different cellular mechanisms is necessary for cell motility, the S100P-induced expression of cathepsin D has been shown to support the migration of Panc-1 cells (25).

In contrast to the highly migrating cell line Panc-1, transwell migration and in vitro wound scratch assays clearly showed a reduced migration of the $\mathrm{H} 358$ cells in response to S100P. This S100P-mediated impact on cell migration was also observed in these cells co-overexpressing RAGE. On the basis of these findings, it is well conceivable that S100P either promotes or limits the migration of cancer cells depending on their origin/subtype. Conflicting data on cell type dependency have also been documented for another S100 protein, namely S100A2 $(5,46)$. Thus, it must be considered that human lung adenocarcinoma represents a heterogeneous group of different histopathological subclasses including bronchiolo-alveolar carcinoma (37). Moreover, lung carcinomas of mixed histology are often diagnosed. As H358 cells correspond to the bronchiolo-alveolar cell type (47), our data also suggest that the less invasive character of bronchiolo-alveolar carcinoma (48) cannot be converted by S100P.

Our data suggest that S100P contributes to early stages in lung adenocarcinoma development, whereas its downregulation in advanced stages might be important for the extensive tumour growth in which transcription changes due to feedback mechanisms and/or DNA methylation play an important role. The simultaneous downregulation of RAGE in lung tumours might not have a significant impact on the S100Pmediated effects in tumour initiation. On the basis of partially divergent observations of other groups, the impact of S100P on tumour progression and metastasis seems to depend on the origin/subtype of cancer cells. In terms of anti-cancer treatment, it will therefore be important to identify the interactive partners of S100P which suppress the tumourpromoting function of S100P in $\mathrm{H} 358$ cells but not in other types of carcinoma cells.

\section{Acknowledgements}

This project was supported by the Deutsche Krebshilfe (107078) and by Wilhelm Roux grants of the Bundesministerium für Bildung und Forschung, BMBF (FKZ 14/14, FKZ 3/30). The authors thank Dr A. Navarrete-Santos and Mrs. S. Koitzsch for the technical support as well as Dr W.D. Schmitt (Institute of Pathology) for the immunohistochemistry.

\section{References}

1. Parkin DM, Bray F, Ferlay J and Pisani P: Global cancer statistics, 2002. CA Cancer J Clin 55: 74-108, 2005.

2. Cersosimo RJ: Lung cancer: a review. Am J Health Syst Pharm 59: 611-642, 2002. 
3. Hofmann HS, Bartling B, Simm A, et al: Identification and classification of differentially expressed genes in non-small cell lung cancer by expression profiling on a global human 59.620element oligonucleotide array. Oncol Rep 16: 587-595, 2006

4. Kim B, Lee HJ, Choi HY, et al: Clinical validity of the lung cancer biomarkers identified by bioinformatics analysis of public expression data. Cancer Res 67: 7431-7438, 2007.

5. Diederichs S, Bulk E, Steffen B, et al: S100 family members and trypsinogens are predictors of distant metastasis and survival in early-stage non-small cell lung cancer. Cancer Res 64: 5564-5569, 2004

6. Bartling B, Rehbein G, Schmitt W, et al: S100A2-S100P expression profile and diagnosis of non-small cell lung carcinoma: impairment by advanced tumour stages and neoadjuvant chemotherapy. Eur J Cancer 43: 1935-1943, 2007

7. Guerreiro Da Silva I, Hu Y, Russo I, et al: S100P calciumbinding protein overexpression is associated with immortalization of human breast epithelial cells in vitro and early stages of breast cancer development in vivo. Int J Oncol 16: 231-241, 2000.

8. Wang G, Platt-Higgins A, Carroll J, et al: Induction of metastasis by S100P in a rat mammary model and its association with poor survival of breast cancer patients. Cancer Res 66: 1199-1207, 2006.

9. Schor AP, Carvalho FM, Kemp C, et al: S100P calcium-binding protein expression is associated with high-risk proliferative lesions of the breast. Oncol Rep 15: 3-6, 2006.

10. Iacobuzio-Donahue CA, Maitra A, Shen-Ong GL, et al: Discovery of novel tumor markers of pancreatic cancer using global gene expression technology. Am J Pathol 160: 1239-1249, 2002.

11. Logsdon CD, Simeone DM, Binkley C, et al: Molecular profiling of pancreatic adenocarcinoma and chronic pancreatitis identifies multiple genes differentially regulated in pancreatic cancer. Cancer Res 63: 2649-2657, 2003.

12. Sato N, Fukushima N, Matsubayashi H and Goggins $M$ : Identification of maspin and S100P as novel hypomethylation targets in pancreatic cancer using global gene expression profiling. Oncogene 23: 1531-1538, 2004.

13. Dowen SE, Crnogorac-Jurcevic T, Gangeswaran R, et al: Expression of S100P and its novel binding partner S100PBPR in early pancreatic cancer. Am J Pathol 166: 81-92, 2005.

14. Mousses S, Bubendorf L, Wagner U, et al: Clinical validation of candidate genes associated with prostate cancer progression in the CWR22 model system using tissue microarrays. Cancer Res 62: 1256-1260, 2002.

15. Hammacher A, Thompson EW and Williams ED: Interleukin-6 is a potent inducer of $\mathrm{S} 100 \mathrm{P}$, which is up-regulated in androgenrefractory and metastatic prostate cancer. Int J Biochem Cell Biol 37: 442-450, 2005.

16. Birkenkamp-Demtroder K, Olesen SH, Sorensen FB, et al: Differential gene expression in colon cancer of the caecum versus the sigmoid and rectosigmoid. Gut 54: 374-384, 2005.

17. Kita H, Hikichi Y, Hikami K, et al: Differential gene expression between flat adenoma and normal mucosa in the colon in a microarray analysis. J Gastroenterol 41: 1053-1063, 2006.

18. Bertram J, Palfner K, Hiddemann W and Kneba M: Elevated expression of S100P, CAPL and MAGE 3 in doxorubicinresistant cell lines: comparison of mRNA differential display reverse transcription-polymerase chain reaction and subtractive suppressive hybridization for the analysis of differential gene expression. Anticancer Drugs 9: 311-317, 1998.

19. Becker T, Gerke V, Kube E and Weber K: S100P, a novel $\mathrm{Ca}^{2+}$ binding protein from human placenta. cDNA cloning, recombinant protein expression and $\mathrm{Ca}^{2+}$ binding properties. Eur J Biochem 207: 541-547, 1992.

20. Jin G, Wang S, Hu X, et al: Characterization of the tissuespecific expression of the s100P gene which encodes an EFhand $\mathrm{C}^{2+}$-binding protein. Mol Biol Rep 30: 243-248, 2003.

21. Koltzscher $M$ and Gerke V: Identification of hydrophobic amino acid residues involved in the formation of S100P homodimers in vivo. Biochemistry 39: 9533-9539, 2000.

22. Donato R: S100: A multigenic family of calcium-modulated proteins of the EF-hand type with intracellular and extracellular functional roles. Int J Biochem Cell Biol 33: 637-668, 2001.

23. Heizmann C, Fritz G and Schäfer B: S100 proteins: structure, functions and pathology. Front Biosci 7: 1356-1368, 2002.

24. Wang G, Zhang S, Fernig DG, et al: Heterodimeric interaction and interfaces of S100A1 and S100P. Biochem J 382: 375-383, 2004.
25. Whiteman HJ, Weeks ME, Dowen SE, et al: The role of S100P in the invasion of pancreatic cancer cells is mediated through cytoskeletal changes and regulation of cathepsin D. Cancer Res 67: 8633-8642, 2007

26. Gribenko AV, Hopper JE and Makhatadze GI: Molecular characterization and tissue distribution of a novel member of the S100 family of EF-hand proteins. Biochemistry 40: 15538-15548, 2001.

27. Koltzscher M, Neumann C, Konig S and Gerke V: $\mathrm{Ca}^{2+}$ dependent binding and activation of dormant ezrin by dimeric S100P. Mol Biol Cell 14: 2372-2384, 2003.

28. Bretscher A, Edwards K and Fehon R: ERM proteins and merlin: integrators at the cell cortex. Nat Rev Mol Cell Biol 3: 586-599, 2002

29. Arumugam T, Simeone DM, Van Golen K and Logsdon CD: S100P promotes pancreatic cancer growth, survival, and invasion. Clin Cancer Res 11: 5356-5364, 2005.

30. Fuentes MK, Nigavekar SS, Arumugam T, et al: RAGE activation by $\mathrm{S} 100 \mathrm{P}$ in colon cancer stimulates growth, migration, and cell signaling pathways. Dis Colon Rectum 50: 1230-1240, 2007.

31. Filipek A, Jastrzebska B, Nowotny M and Kuznicki J: CacyBP/ SIP, a calcyclin and Siah-1-interacting protein, binds EF-hand proteins of the S100 family. J Biol Chem 277: 28848-28852, 2002.

32. Nowotny M, Spiechowicz M, Jastrzebska B et al: Calciumregulated interaction of Sgt1 with S100A6 (calcyclin) and other S100 proteins. J Biol Chem 278: 26923-26928, 2003.

33. Arumugam T, Simeone D, Schmidt A and Logsdon C: S100P stimulates cell proliferation and survival via receptor for activated glycation end products (RAGE). J Biol Chem 279: 5059-5065, 2004

34. Donato R: Intracellular and extracellular roles of S100 proteins. Microsc Res Tech 60: 540-551, 2003.

35. Hofmann H-S, Hansen G, Burdach S, et al: Classification of human lung neoplasm by two target genes. Am J Respir Crit Care Med 170: 516-519, 2004.

36. Bartling B, Hofmann H, Weigle B, et al: Down-regulation of the receptor for advanced glycation end-products (RAGE) supports non-small cell lung carcinoma. Carcinogenesis 26 : 293-301, 2005.

37. Travis WD, Brambilla E, Müller-Hermelink HK and Harris C: WHO classification of tumors. Pathology and genetics: Tumors of the lung, pleura, thymus and heart. IARC Press, Lyon, 2004.

38. Dell'Orco RT, Mertens JG and Kruse PF Jr: Doubling potential, calendar time, and senescence of human diploid cells in culture. Exp Cell Res 77: 356-360, 1973.

39. Lawrence DA, Pircher R, Kryceve-Martinerie C and Jullien P. Normal embryo fibroblasts release transforming growth factors in a latent form. J Cell Physiol 121: 184-188, 1984.

40. Wang Q, Williamson M, Bott S, et al: Hypomethylation of WNT5A, CRIP1 and S100P in prostate cancer. Oncogene 26: 6560-6565, 2007

41. Takahashi T, Carbone D, Nau MM, et al: Wild-type but not mutant p53 suppresses the growth of human lung cancer cells bearing multiple genetic lesions. Cancer Res 52: 2340-2343, 1992.

42. Crnogorac-Jurcevic T, Missiaglia E, Blaveri E, et al: Molecular alterations in pancreatic carcinoma: expression profiling shows that dysregulated expression of S100 genes is highly prevalent. J Pathol 201: 63-74, 2003.

43. Ohuchida K, Mizumoto K, Egami T, et al: S100P is an early developmental marker of pancreatic carcinogenesis. Clin Cancer Res 12: 5411-5416, 2006

44. Gibadulinova A, Barathova M, Kopacek J, et al: Expression of $\mathrm{S} 100 \mathrm{P}$ protein correlates with and contributes to the tumorigenic capacity of HeLa cervical carcinoma cells. Oncol Rep 14: 575-582, 2005.

45. Missiaglia E, Blaveri E, Terris B, et al: Analysis of gene expression in cancer cell lines identifies candidate markers for pancreatic tumorigenesis and metastasis. Int J Cancer 112: 100-112, 2004.

46. Nagy N, Brenner C, Markadieu N, et al: S100A2, a putative tumor suppressor gene, regulates in vitro squamous cell carcinoma migration. Lab Invest 81: 599-612, 2001.

47. Schuller H, del Campo A, McMahon J, et al: Ultrastructural classification of human lung cancer cell lines as a basis to study the response of different types of lung tumors to chemicals and the drugs in vitro. Proc Am Assoc Cancer Res 26: 27, 1985.

48. Raz DJ, He B, Rosell R and Jablons DM: Bronchioloalveolar carcinoma: a review. Clin Lung Cancer 7: 313-322, 2006. 